\title{
Casimir energy for a scalar field with a frequency dependent boundary condition
}

\author{
H. Falomir and K. Rébora \\ IFLP, Departamento de Física, Facultad de Ciencias Exactas, Universidad Nacional de La Plata, C.C. 67, (1900) La Plata, Argentina \\ M. Loewe \\ Facultad de Física, Pontificia Universidad Católica de Chile, Casilla 306, Santiago 22, Chile
}

(Received 31 August 2000; published 22 December 2000)

\begin{abstract}
We consider the vacuum energy for a scalar field subject to a frequency dependent boundary condition. The effect of a frequency cutoff is described in terms of an incomplete $\zeta$ function. The use of the Debye asymptotic expansion for Bessel functions allows us to determine the dominant (volume, area, ... ) terms in the Casimir energy. The possible interest of this kind of model for dielectric media (and its application to sonoluminescence) is also discussed.
\end{abstract}

DOI: 10.1103/PhysRevD.63.025015

PACS number(s): 12.20.Ds, 03.70.+k, 78.60.Mq

\section{INTRODUCTION}

The Casimir effect [1,2] arises as a distortion of the vacuum energy of quantized fields due to the presence of boundaries (or nontrivial topologies) in the quantization domain. This effect, which has a quantum nature associated with the zero-point oscillations in the vacuum state, is significant in diverse areas of physics, from statistical physics to elementary particle physics and cosmology.

In particular, in the last few years there has been great interest in the Casimir energy of electromagnetic fields in the presence of dielectric media, due to Schwinger's suggestion [3] that it could play a role in the explanation of the phenomenon of sonoluminescence [4].

The results obtained on this subject by different groups through several calculation techniques (such as Green's functions methods, van der Waals forces, $\zeta$-function methods, and asymptotic developments for the density of statessee Refs. [5-15], among others) are rather controversial, and some basic issues remain to be clarified. In this respect, it is our aim to contribute to the understanding of the problem by studying a simplified model which incorporates a frequency cutoff in the boundary conditions at the separation between the media in order to emulate the behavior of real dielectrics.

It should be mentioned that authors introducing a cutoff in the wave number to describe the behavior of real dielectrics agree with Schwinger's explanation of the phenomenon (see, for example, [11-14]). On the other hand, in Ref. [15], where the permittivity of a nonmagnetic medium is modeled by means of a (angular momentum dependent) oneabsorption-frequency Sellmeir relation, and a $\zeta$-function technique is employed to sum up the contributions of the proper oscillation modes of the electromagnetic field, it is concluded that the vacuum energy of a bubble embedded in this material has the wrong behavior with the radius (in addition to its absolute value being far too small) to be relevant to sonoluminescence.

Candelas [16] has been the first to remark on the importance of dispersion in connection with the Casimir effect. Later, Brevik and collaborators [17-20] have considered several models with spherical and cylindrical geometries, showing that dispersive effects may reverse the direction of the Casimir force acting on the boundary between media. In Refs. $[17,18]$ the case of a spherically symmetric media satisfying the relation $\epsilon(\omega) \mu(\omega)=1$ is studied (of special interest in QCD), with both a sharp frequency cutoff on $\mu(\omega)$ and a behavior analogous to the one-absorption-frequency Sellmeir's formula for dielectrics. In Ref. [19], for nonmagnetic dispersive media with spherical geometry, and based upon the Minkowski energy-momentum tensor, the Casimir surface force is worked out when the permittivity $\epsilon(\omega)$ presents a step along the imaginary frequency axis. In the limit of perfect conductivity, a nondispersive term is recovered, which is in agreement with Boyer result [21,22], while an attractive dispersive contribution is also found.

In what follows we consider a simple model of a scalar field subject to frequency dependent local boundary conditions on the surface of a sphere. Thus our main goal is to establish a method for calculating the change of the Casimir energy of the field when the radius of the sphere is varied, in a situation where the boundary conditions impose a physical frequency cutoff $\Omega$.

To this end we consider the very simple case of a scalar field whose modes corresponding to eigenfrequencies $\omega$ $\leqslant \Omega$ are confined to the interior of a sphere of radius $R$, satisfying local homogeneous boundary conditions.

On the other hand, we will assume that the boundary is completely transparent for those modes with $\omega>\Omega$. Therefore, their contribution to the difference in Casimir energies for two different values of $R$ will cancel out, no matter the regularization employed for its definition. Consequently, we will subtract these contributions, which amounts to a redefinition of the reference energy level in an $R$-independent way.

For the evaluation of the vacuum energy of the low frequency modes we will employ asymptotic expansions in an incomplete- $\zeta$ summation technique, to be discussed in the following. This approach will allow for the identification of the volume, surface, ..., dominant terms in the Casimir energy.

As a final exercise we will show that, with reasonable values for the frequency cutoff and the bubble radius, the 
amount of Casimir energy available in this model is comparable with the energy emitted during each cycle of a typical sonoluminescence experiment [4]. In view of the controversy existing on the subject, it would be of great interest to apply this method to a similar model for the case of the electromagnetic field in the presence of dielectric media, a calculation which will be presented in a forthcoming paper [23].

\section{THE MODEL AND ITS INCOMPLETE $\zeta$ FUNCTION}

Let us consider a free scalar field in $\mathbf{R}^{3}$ satisfying local boundary conditions which depend on the frequency $\omega$ of the field modes at the surface of a sphere of radius $R$.

We will make the assumption that the boundary is completely transparent for the modes of frequencies greater than a cutoff $\Omega$, while for $\omega \leqslant \Omega$ the modes satisfy Dirichlet boundary conditions

$$
\begin{gathered}
\left(\Delta+\frac{\omega^{2}}{c^{2}}\right) \psi_{\omega}(\vec{r})=0, \quad \text { for } r<R, \\
\left.\psi_{\omega}(\vec{r})\right|_{r=R}=0,
\end{gathered}
$$

being confined to the interior of the sphere.

Writing $\psi_{\omega}(\vec{r})=f_{l}(r) Y_{l}^{m}(\theta, \varphi)$, we get for the radial function

$$
\left(\frac{d^{2}}{d r^{2}}+\frac{2}{r} \frac{d}{d r}-\frac{l(l+1)}{r^{2}}+\frac{\omega^{2}}{c^{2}}\right) f_{l}(r)=0, \quad \text { for } r<R,
$$

where the eigenfrequencies are determined by imposing the condition

$$
\left.f_{l}(r)\right|_{r=R}=0 .
$$

The solutions that are regular at the origin are given by $f_{l}(r)=\sqrt{\pi / 2 z} J_{\nu}(z)$, with $\nu=l+1 / 2$ and $z=\omega_{\nu, n} r / c$, where the eigenfrequencies are

$$
\omega_{\nu, n}=\frac{c}{R} j_{\nu, n},
$$

$j_{\nu, n}$ being the $n$th zero of the Bessel function $J_{\nu}(z)$.

We will be interested in the difference between the vacuum energies of two situations differing in the value of $R$. Then, we can disregard the contributions of those modes with frequencies $\omega>\Omega$ because, being independent of the position of the boundary, their contributions cancel out (whatever the regularization employed in defining the vacuum energy would be). This simply amounts to an $R$-independent subtraction, which is nothing but a redefinition of the zero energy level.

Therefore, we should evaluate the (finite) sum

$$
E(R)=\sum_{\nu=1 / 2}^{\nu_{0}} 2 \nu \sum_{n=1}^{N_{\nu}} \frac{1}{2} \hbar \omega_{\nu, n},
$$

where $N_{\nu}$ is the number of positive zeros of $J_{\nu}(z)$ less than or equal to $x=\Omega R / c$, the factor $2 \nu=2 l+1$ is the eigenvalue degeneracy, and $\nu_{0}$ is the maximum value of $\nu$ for which $N_{\nu} \geqslant 1$.

We are interested in an analytic, rather than numeric, evaluation of Eq. (5). Although this is a finite sum, we will employ a summation method based on the evaluation of an incomplete $\zeta$ function, an approach which could be applied in more complex situations. We can employ the following representation:

$$
\sum_{n=1}^{N_{\nu}} j_{\nu, n}=\left.\sum_{n=1}^{N_{\nu}} j_{\nu, n}^{-s}\right|_{s=-1},
$$

where the sum in the right-hand side obviously exists for any $s \in \mathbf{C}{ }^{1}$

Since $J_{\nu}(z)$, for $\nu>-1$, has only real zeros, and its nonvanishing zeros are all simple [24], we can employ the Cauchy theorem to represent the sum in the right-hand side of Eq. (6) as an integral on the complex plane,

$$
\sum_{n=1}^{N_{\nu}} j_{\nu, n}^{-s}=\frac{1}{2 \pi \imath} \oint_{C} z^{-s} \frac{J_{\nu}^{\prime}(z)}{J_{\nu}(z)} d z,
$$

where the curve $C$ encircles the $N_{\nu}$ first positive zeros of $J_{\nu}(z)$ counterclockwise.

For large enough $\mathfrak{R}(s)$, the contour $C$ can be deformed into two straight vertical lines, one crossing the horizontal axis at $\mathfrak{R}(z)=x$ and the other at $\mathfrak{R}(z)=0^{+}$. Indeed, expressing the integrand in terms of the modified Bessel function [25]

$$
I_{\nu}(w)=e^{-\imath(\pi / 2) \nu} J_{\nu}\left(e^{i(\pi / 2)} w\right)
$$

(valid for $-\pi<\arg (w) \leqslant \pi / 2$ ), and taking into account its asymptotic behavior for large arguments [25], it is easily seen that, for $0<x \neq j_{\nu, n}, \forall n$, the integral

$$
\zeta_{\nu}(s, x) \equiv \frac{-1}{2 \pi l} \int_{x-l^{\infty}}^{x+l^{\infty}} z^{-s} \frac{J_{\nu}^{\prime}(z)}{J_{\nu}(z)} d z
$$

converges absolutely and uniformly in the open half-line $s$ $>1$, from which it can be meromorphically extended to the whole complex $s$ plane.

Therefore, for $s>1$,

$$
\sum_{n=1}^{N_{\nu}} j_{\nu, n}^{-s}=\zeta_{\nu}\left(s, 0^{+}\right)-\zeta_{\nu}(s, x)
$$

And, since the left-hand side of Eq. (10) is holomorphic in $s$, the singularities of $\zeta_{\nu}(s, x)$ must be independent of $x$.

On the other hand, for $y>0$ [25],

\footnotetext{
${ }^{1}$ Notice that the sum in the right-hand side of Eq. (6) evaluated at $s=0$ gives $N_{\nu}$, the number of eigenfrequencies contributing to the Casimir energy of the field (after the subtraction made to define it) for a given value of the angular momentum $l=\nu-1 / 2$.
} 


$$
\begin{gathered}
I_{\nu}(-y-i x)=e^{-\imath \pi \nu} I_{\nu}(y+i x), \\
I_{\nu}(y+i x)=\left(I_{\nu}(y-i x)\right) * .
\end{gathered}
$$

So, for real $s>1$ we can write

$$
\begin{aligned}
\zeta_{\nu}(s, x)= & \Re\left\{\frac{-x^{1-s}}{\pi} e^{-i(\pi \cdot 2)(s+1)}\right. \\
& \left.\times \int_{0}^{\infty}(y-\imath)^{-s} \frac{I_{\nu}^{\prime}(x(y-\imath))}{I_{\nu}(x(y-\imath))} d y\right\} .
\end{aligned}
$$

In order to construct the analytic extension of $\zeta_{\nu}(s, x)$ to $s \simeq-1$, we subtract and add to the integrand in Eq. (12) the first few terms obtained from the uniform asymptotic (Debye) expansion [25] of the Bessel functions,

$$
\frac{I_{\nu}^{\prime}(\nu t)}{I_{\nu}(\nu t)}=\frac{1}{\nu} D_{\nu}(t)+\mathcal{O}\left(\nu^{-3}\right)
$$

where

$$
\begin{aligned}
D_{\nu}(t) & =\nu D^{(1)}(t)+D^{(0)}(t)+\nu^{-1} D^{(-1)}(t) \\
& =\frac{\nu \sqrt{1+t^{2}}}{t}-\frac{t}{2\left(1+t^{2}\right)}+\frac{4 t-t^{3}}{8 \nu\left(1+t^{2}\right)^{5 / 2}}
\end{aligned}
$$

is valid for large $\nu$ with fixed $t$. We will see that this approximation allows for the identification of the volume, surface, ..., contributions to the vacuum energy.

Changing the integration variable in Eq. (12) to $t \equiv z(y$ $-\imath$ ), with $z=x / \nu>0$, we get

$\zeta_{\nu}(s, x)=\Re\left\{\frac{-\nu^{-s}}{\pi} e^{-i(\pi / 2)(s+1)} \int_{-i z}^{\infty-i z} t^{-s} \frac{d\left(\ln I_{\nu}(\nu t)\right)}{d t} d t\right\}$.

So, we must consider the integral

$$
\begin{aligned}
\int_{-i z}^{\infty-i z} t^{-s} \frac{d\left(\ln I_{\nu}(\nu t)\right)}{d t} d t= & \int_{-i z}^{\infty-i z} t^{-s} D_{\nu}(t) d t \\
& +\int_{-i z}^{\infty-i z} t^{-s}\left\{\frac{d\left(\ln I_{\nu}(\nu t)\right)}{d t}\right. \\
& \left.-D_{\nu}(t)\right\} d t .
\end{aligned}
$$

The second integral in the right-hand side of this equation converges for $s>-2$, since the integrand can be estimated by means of the next $\left(\mathcal{O}\left(\nu^{-3}\right)\right)$ term in the Debye expansion [Eq. (13)], which behaves as $\mathcal{O}\left(t^{-3}\right)$ for large $|t|$. It can be numerically evaluated at $s=-1$. This will not be done in this paper.

In the following, we will consider only the first integral in the right-hand side of Eq. (16), retaining the first few terms of its expansion in powers of $\nu^{-1}$, consistent with the approximation made in Eq. (13). Notice that the integrand is an algebraic function, having singularities at $t=0, \pm l$, and be- having as $\mathcal{O}\left(t^{0}\right)$ for large $|t|$. This integral converges absolutely and uniformly for $s>1$, where it defines an analytic function to be meromophically extended to the region of interest of the parameter $s$. As we will see, this extension reveals the singularities of $\zeta(s, x)$ as simple poles, whose residues are independent of $x$ [a necessary condition to give a finite result in Eq. (10) for any $s$ ].

In fact, by virtue of the analyticity of the integrand, we can change the path of integration to write

$$
\begin{aligned}
\int_{-i z}^{\infty-i z} & t^{-s} D_{\nu}(t) d t \\
= & \int_{-i z}^{1} t^{-s}\left(\frac{\nu \sqrt{1+t^{2}}}{t}-\frac{t}{2\left(1+t^{2}\right)}+\frac{4 t-t^{3}}{8 \nu\left(1+t^{2}\right)^{5 / 2}}\right) d t \\
& +\int_{1}^{\infty} t^{-s}\left\{\frac{\nu \sqrt{1+t^{2}}}{t}-\frac{t}{2\left(1+t^{2}\right)}+\frac{4 t-t^{3}}{8 \nu\left(1+t^{2}\right)^{5 / 2}}\right. \\
& \left.-\left[\nu\left(1+\frac{1}{2 t^{2}}\right)-\frac{1}{2 t}-\frac{1}{8 \nu t^{2}}\right]\right\} d t \\
& +\int_{1}^{\infty} t^{-s}\left[\nu\left(1+\frac{1}{2 t^{2}}\right)-\frac{1}{2 t}-\frac{1}{8 \nu t^{2}}\right] d t .
\end{aligned}
$$

The first integral in the right-hand side of Eq. (17), containing the whole dependence on $x=\nu z$, is holomorphic in $s$ and can be directly evaluated at the required value of this parameter. On the half-line $(1, \infty)$ we have subtracted and added the first terms in the series expansion of $D_{\nu}(t)$ for large $t$, thus making the second integral to converge for $s>$ -2 . The third one must be evaluated for $s>1$ and then analytically continued to the relevant values of $s$. This can be done exactly, its contribution to $\zeta_{\nu}(s, x)$ in Eq. (15) being the real part of

$$
\frac{e^{-(i / 2) \pi(1+s)}}{\nu^{1+s} 8 \pi}\left(\frac{8 \nu^{2}}{1-s}+\frac{4 \nu}{s}+\frac{1-4 \nu^{2}}{1+s}\right)
$$

This expression has simple poles at $s=0, \pm 1$, which are the only singularities of $\zeta_{\nu}(s, x)$ for $\mathfrak{R}(s)>-2$. Notice that the residues of $\zeta_{\nu}(s, x)$ are independent of $x$,

$$
\begin{gathered}
\left.\operatorname{Res} \zeta_{\nu}(s, x)\right|_{s=1}=\frac{1}{\pi}, \\
\left.\operatorname{Res} \zeta_{\nu}(s, x)\right|_{s=0}=0, \\
\left.\operatorname{Res} \zeta_{\nu}(s, x)\right|_{s=-1}=\frac{1-4 \nu^{2}}{8 \pi},
\end{gathered}
$$

and in agreement with the results in [26] [where $\zeta_{\nu}\left(s, 0^{+}\right)$is studied].

For example, for $\zeta_{\nu}(s, x)$ around $s=-1$ and for $\nu<x$ (which will be needed in Sec. IV to evaluate the vacuum energy), one straightforwardly obtains the Laurent expansion 


$$
\begin{aligned}
& \zeta_{\nu}(s, x>\nu)=\frac{1-4 \nu^{2}}{8 \pi(1+s)}+\left[\frac { ( 4 \nu ^ { 2 } - 1 ) } { 8 \pi } \left[\log \left(\frac{\nu}{2}\right)\right.\right. \\
&\left.+\log \left(z+\sqrt{z^{2}-1}\right)\right]-\frac{\nu^{2}}{4 \pi}-\frac{\nu^{2}}{2 \pi} z \sqrt{z^{2}-1} \\
&\left.\quad-\frac{3 z-8 z^{3}}{24 \pi\left(z^{2}-1\right)^{3 / 2}}-\frac{1}{3 \pi}+\mathcal{O}\left(\nu^{-1}\right)\right]+\mathcal{O}(s+1),
\end{aligned}
$$

with fixed $z=x / \nu \geq 1$.

On the other hand, for $x \rightarrow 0^{+}$a similar calculation leads to

$$
\begin{aligned}
\zeta_{\nu}\left(s, x=0^{+}\right)= & \frac{1-4 \nu^{2}}{8 \pi(1+s)}+\left[\frac{\left(4 \nu^{2}-1\right)}{8 \pi} \log \left(\frac{\nu}{2}\right)-\frac{\nu^{2}}{4 \pi}-\frac{\nu}{4}\right. \\
& \left.-\frac{1}{3 \pi}+\mathcal{O}\left(\nu^{-1}\right)\right]+\mathcal{O}(s+1) .
\end{aligned}
$$

In the following section we will evaluate, as a function of $\nu$, the number of modes contributing in Eq. (10), and in Sec. IV, their contributions to the vacuum energy.

\section{THE NUMBER OF CONTRIBUTING MODES}

In this section we address ourselves to the determination of $\nu_{0}$ in Eq. (5), the maximum value of $\nu$ for which $N_{\nu}$ $\geqslant 1$. Although in the simple case under study the zeros of $J_{\nu}(w)$ are well known [25], we prefer to establish a criterium which can be applied in more general situations.

First, notice that

$$
\left.N_{\nu}(x) \equiv \sum_{n=1}^{N_{\nu}} j_{\nu, n}^{-s}\right|_{s=0}=\left.\left[\zeta_{\nu}\left(s, 0^{+}\right)-\zeta_{\nu}(s, x)\right]\right|_{s=0}
$$

is a discontinuous function of $x$, having a step of height one at each positive zero $j_{\nu, n}$ of the Bessel function $J_{\nu}(w)$.

Then, $\nu_{0}$ can be determined from the condition

$$
N_{\nu_{0}}(x)=N_{\nu_{0}}\left(j_{\nu_{0}, 1}+0\right)=1 \text {, }
$$

with $N_{\nu_{0}}\left(j_{\nu_{0}, 1}-0\right)=0$.

Taking into account Eq. (19) and the fact that the second and third integrals in the right-hand side of Eq. (17) are real, it is straightforward to obtain from Eqs. (15)-(17) that

$$
\begin{aligned}
\zeta_{\nu}(s=0, x)= & -\frac{\nu}{2}-\frac{1}{4}+\mathfrak{R}\left\{-\frac{l \nu}{\pi}\left(\sqrt{1+e^{-l \pi} z^{2}}\right.\right. \\
& \left.-\log \left(1+\sqrt{1+e^{-l \pi} z^{2}}\right)\right)+\frac{l}{4 \pi} \log \left(1+e^{-\imath \pi} z^{2}\right) \\
& \left.+\frac{l\left(2+3 z^{2}\right)}{24 \nu \pi\left(1+e^{-\imath \pi} z^{2}\right)^{3 / 2}}\right\}+\mathcal{O}\left(\nu^{-2}\right)
\end{aligned}
$$

where we have taken $z=x / \nu \approx 1$. In particular, for $x \rightarrow 0^{+}$,

$$
\zeta_{\nu}\left(s=0, x=0^{+}\right)=-\frac{\nu}{2}-\frac{1}{4}+\mathcal{O}\left(\nu^{-2}\right),
$$

is a coincidence with [26].

Now, taking the difference between Eqs. (25) and (24) we get a smooth approximation, $\widetilde{N}_{\nu}(x)+\mathcal{O}\left(\nu^{-1}\right)$, to the step function $N_{\nu}(x)$ in Eq. (22). It is easily seen that, for $\nu>x$, $\tilde{N}_{\nu}(x)=0$ while, for $\nu<x$, we have

$$
\begin{aligned}
\tilde{N}_{\nu}(x)= & \frac{\nu}{\pi}\left(\sqrt{z^{2}-1}-\arctan \left(\sqrt{z^{2}-1}\right)\right)-\frac{1}{4} \\
& -\frac{2+3 z^{2}}{24 \nu \pi\left(z^{2}-1\right)^{3 / 2}},
\end{aligned}
$$

with $z=x / \nu$.

Let us now determine the value $\nu_{0}$ for which

$$
\tilde{N}_{\nu_{0}}(x)=1 / 2 \text {. }
$$

To this end, we propose an expansion of the form

$$
\sqrt{z_{0}^{2}-1}=\varepsilon_{1} \nu_{0}^{-1 / 3}+\varepsilon_{3} \nu_{0}^{-3 / 3}+\mathcal{O}\left(\nu_{0}^{-5 / 3}\right),
$$

which makes sense for $\nu_{0} \gg 1$ and $z_{0}=x / \nu_{0} \approx 1$. Replacing in Eq. (26) and imposing Eq. (27), the coefficients $\varepsilon_{k}$ can be determined order by order in $\nu_{0}^{-1 / 3}$ to get

$$
x=\nu_{0}+1.857 \nu_{0}^{1 / 3}+1.034 \nu_{0}^{-1 / 3}+\mathcal{O}\left(\nu_{0}^{-1}\right)
$$

or, inverting this development,

$$
\nu_{0}=x-1.857 x^{1 / 3}+0.1155 x^{-1 / 3}+\mathcal{O}\left(x^{-1}\right) .
$$

(Notice that $\nu_{0}<x$.)

Equation (29) is in excellent agreement with the expression of the first nonvanishing zero of $J_{\nu_{0}}(w)$, for a large order $\nu_{0}$ [25]: $j_{\nu_{0}, 1}=\nu_{0}+1.8557 \nu_{0}^{1 / 3}+1.03315 \nu_{0}^{-1 / 3}$ $+\mathcal{O}\left(\nu_{0}^{-1}\right)$.

\section{THE DOMINANT CONTRIBUTIONS TO THE VACUUM ENERGY}

In this section we evaluate the first contributions to the vacuum energy obtained from the Debye expansion employed in Sec. II. As we will see, this allows us to determine the volume, surface, ..., terms in the Casimir energy of the scalar field.

According to the results in the previous section, we are interested in the Laurent expansion of $\zeta_{\nu}(s, x>\nu)$ and $\zeta_{\nu}\left(s, x=0^{+}\right)$around $s=-1$, given in Eqs. (20) and (21). As already remarked, the singular parts cancel out in the difference on the right-hand side of Eq. (10) [see Eq. (19)]. For the difference of the finite parts we get

$$
\begin{aligned}
z F(x, \nu) \equiv & {\left[\zeta_{\nu}\left(s, 0^{+}\right)-\zeta_{\nu}(s, x)\right]_{s=-1} } \\
= & \frac{\nu^{2}}{2 \pi}\left(z \sqrt{-1+z^{2}}-\log \left(z+\sqrt{-1+z^{2}}\right)\right)-\frac{\nu}{4} \\
& +\frac{3 z-8 z^{3}}{24 \pi\left(-1+z^{2}\right)^{3 / 2}}+\frac{1}{8 \pi} \log \left(z+\sqrt{-1+z^{2}}\right) \\
& +\mathcal{O}\left(\nu^{-1}\right) .
\end{aligned}
$$

This is a good approximation as long as $\nu \gg 1$ and $z=x / \nu$ $\gtrsim 1$. 
Our aim is now to evaluate the sum in Eq. (5),

$$
\begin{aligned}
E(R=x c / \Omega) & =\frac{\hbar \Omega}{x} \sum_{\nu=1 / 2}^{\nu_{0}} \nu\left[\zeta_{\nu}(-1, x)-\zeta_{\nu}\left(-1,0^{+}\right)\right] \\
& =\hbar \Omega \sum_{\nu=1 / 2}^{\nu_{0}} F(x, \nu),
\end{aligned}
$$

with $\nu_{0}$ given in Eq. (30).

The function $F(x, \nu)$ is non-negative and has a pronounced maximum at $\nu \approx x / 2$ (i.e., $z \approx 2$ ). Thus, the use of the approximation in Eq. (31) is consistent if $x \gg 1$.

From Eq. (31), it is not difficult to see that the successive terms in the Euler-Maclaurin summation formula [25],

$$
\begin{aligned}
\sum_{\nu=1 / 2}^{\nu_{k}} F(x, \nu)= & \int_{1 / 2}^{\nu_{0}} F(x, \nu) d \nu+\frac{1}{2}\left\{F\left(x, \nu_{0}\right)+F(x, 1 / 2)\right\} \\
& +\left.\frac{1}{12} \partial_{\nu}[F(x, \nu)]\right|_{\nu=1 / 2} ^{\nu=\nu_{0}}+\ldots
\end{aligned}
$$

are of increasing order in $x^{-1}$. So, retaining the first few terms consistent with the approximation made, we get for the vacuum energy

$$
\begin{aligned}
\frac{E(R)}{\hbar \Omega}= & x^{3}\left(\frac{-\left(\sqrt{-1+z_{0}^{2}}\left(-5+2 z_{0}^{2}\right)\right)}{24 \pi z_{0}^{3}}\right. \\
& \left.+\frac{2 z_{0}^{4}-3 \log \left(z_{0}+\sqrt{-1+z_{0}^{2}}\right)}{24 \pi z_{0}^{4}}\right)-x^{2}\left(\frac{-\sqrt{-1+z_{0}^{2}}}{4 \pi z_{0}^{2}}\right. \\
& \left.+\frac{\pi+3 \log \left(z_{0}+\sqrt{-1+z_{0}^{2}}\right)}{12 \pi z_{0}^{3}}\right)-x\left(\frac{-\left(-27+17 z_{0}^{2}\right)}{48 \pi z_{0} \sqrt{-1+z_{0}^{2}}}\right. \\
& \left.+\frac{6 \pi-10 z_{0}^{2}+69 \log \left(z_{0}+\sqrt{-1+z_{0}^{2}}\right)}{48 \pi z_{0}^{2}}\right)+\mathcal{O}\left(x^{0}\right),
\end{aligned}
$$

where $z_{0}=x / \nu_{0}, x=R \Omega / c$, and $\nu_{0}$ is given in Eq. (30). In this equation one can recognize volume, surface, and curvature contributions to $E(R)$. Notice that we could have retained any number of terms of the asymptotic expansion in Eq. (13) and performed the same steps as in the present calculation to get the Casimir energy to any order in $x^{-1}$.

Finally, replacing in Eq. (34) $z_{0} \rightarrow x / \nu_{0}$ and $\nu_{0}$ by its expression, we get

$$
E(R)=\hbar \Omega\left[\frac{x^{3}}{12 \pi}-\frac{x^{2}}{12}-0.1343 x^{4 / 3}+\mathcal{O}(x)\right]
$$

where one can see that the volume and surface terms are dominant for $x \gg 1$. Also notice that noninteger powers of the radius $R$ appear as a consequence of the relation between $\nu_{0}$ and $x$, Eq. (30).

\section{CONCLUSIONS}

In Eqs. (34)-(35) we have derived the dominant contributions to the vacuum energy of a scalar field in a model with a frequency dependent boundary condition, consisting in the confinement of the modes with low frequency (up to a physical cutoff $\Omega$ ) to the interior of a sphere of radius $R$.

These modes are subject to Dirichlet boundary conditions at the surface of the sphere, while those with frequencies higher than $\Omega$ are free, being that the boundary is completely transparent to them. This characteristic of the model allows for the subtraction of the contribution of the high frequency modes to the vacuum energy, which amounts (independently of the regularization employed to define it) to an $R$-independent redefinition of the zero energy level, having no consequences on the evaluation of energy differences.

In so doing, we have represented the sum over the eigenfrequencies up to the cutoff $\Omega$ in terms of an incomplete $\zeta$ function associated with the Laplacian operator in the sphere with Dirichlet boundary conditions [see Eq. (10)]. The function $\zeta(s, x)$, as in Eq. (12), is well defined only for $\mathfrak{R}(s)$ $>1$. So, it was analytically continued from $s>1$ to the relevant values of this parameter $(s=0$, needed to evaluate the maximum angular momentum $l_{0}=\nu_{0}-1 / 2$, giving rise to eigenfrequencies less than or equal to $\Omega$, and $s=-1$, necessary to evaluate the contribution to the Casimir energy of the modes with angular momentum $l=\nu-1 / 2$ ) by approximating the behavior of the integrand in Eq. (12), employing the Debye asymptotic expansion of the modified Bessel functions appearing in its expression.

This procedure has lead to a meromorphic function having simple poles with (exactly evaluated) cutoff independent residues [see Eq. (19)], a necessary condition to have a finite result for the sum in Eq. (10).

The finite part of $\zeta(s, x)$ has been evaluated up to terms of a given order in $\nu^{-1}$. Although we have retained only the first terms in this asymptotic expansion, one can follow the same steps to determine the finite part of $\zeta(s, x)$ at $s=-1$ or 0 up to any required precision in $\nu^{-1}$.

Finally, the application of the Euler-Maclaurin summation formula has lead to an expression for the Casimir energy of the model in which one can recognize volume, surface, and curvature contributions [see Eq. (34)].

For a cutoff corresponding to $x=R \Omega / c \gg 1$, the dominant terms in the vacuum energy, Eq. (35), are proportional to the volume $\left(V=4 \pi R^{3} / 3\right)$ and area $\left(S=4 \pi R^{2}\right)$ of the sphere,

$$
\frac{E(R)}{\hbar \Omega}=V \frac{\Omega^{3}}{16 \pi^{2} c^{3}}+\xi S \frac{\Omega^{2}}{12 \pi^{2} c^{2}}+\cdots,
$$

with $\xi=-\pi / 4$.

It is worthwhile to remark that, for a similar model where the low frequency modes of the scalar field are subject to Neumann (rather than Dirichlet) boundary conditions, we get the same expression for the dominant terms with $\xi=+\pi / 4$.

These two dominant terms are in complete agreement with those obtained from the expansion of the density of 
states in powers of the inverse wavelength ${ }^{2}[27,11]$. The relation between $\nu_{0}$ and $x$, Eq. (30) (or, equivalently, the expression of the first zero of the Bessel function $J_{\nu_{0}}(w)$ in terms of the order $\nu_{0}[25]$ ) also introduces noninteger powers of the radius $R$ [see Eq. (35)].

As a final exercise, we can use Eq. (35) in a very schematic model pretending to mimic the phenomenon of sonoluminescence. We will adopt the values of the radius and emitted energy corresponding to a typical sonoluminescent bubble [4], and estimate the cutoff $\Omega$ needed to produce this amount of energy. To this end, we will simply take the difference of the low frequency contribution to the Casimir en-

${ }^{2}$ Indeed, for scalar fields subject to local homogeneous boundary conditions the density of states is modified by finite volume effects [27]. The first correction in the asymptotic expansion for large wavelength $k$ is given by

$$
\sum_{n} \sim V \int \frac{d^{3} k}{(2 \pi)^{3}}+S \int \xi \frac{d^{3} k}{(2 \pi)^{3} k}+\ldots,
$$

where the coefficient $\xi$ takes the value $\xi=-\pi / 4, \xi=+\pi / 4$, and $\xi=+\pi / 4$ for Dirichlet, Neumann, and Robin boundary conditions, respectively. Then, introducing the dispersion relation $\omega(k)=c k$ and a cutoff in the wavelength given by $K=\Omega / c$, it is easy to get Eq. (36) for the vacuum energy. For junction boundary conditions, as those appearing in problems with dielectrics, the coefficient $\xi$ has more involved expressions, which can be difficult to evaluate [11]. ergy of the scalar field for two different values of the bubble radius.

If the bubble collapses from an initial radius $R=4$ $\times 10^{-5} \mathrm{~m}$ to a final radius of one tenth this value, and the emitted energy is $\mathcal{E}=1.2 \times 10^{-12} \mathrm{~J}$, by imposing the equality

$$
\frac{R}{\hbar c}(E(R)-E(R / 10))=1.516 \times 10^{9},
$$

and taking into account Eq. (35), it follows that $x=490$, justifying the use of the approximation obtained.

This implies that $\Omega=3.675 \times 10^{15} 1 / \mathrm{sec}$, which corresponds to a cutoff in wavelengths in the ultraviolet of $\Lambda$ $=5.129 \times 10^{-7} \mathrm{~m}=5129 \AA$, not far from the region where the refraction index of water essentially becomes one [28]. This strongly suggests that we consider a similar model for the case of the electromagnetic field in the presence of dielectric media, a calculation which will be presented elsewhere [23].

\section{ACKNOWLEDGMENTS}

The authors thank E.M. Santangelo for useful comments. This work was partially supported by Fundaciones AndesAntorchas under Contract No. C-13398/6, ANPCyT (Argentina)-PICT'97 No. 00039, CONICET (Argentina)-PIP No. 0459, and FONDECyT (Chile)-grants Nos. 1980577 and 7980011.
[1] H. B. G. Casimir, Proc. K. Ned. Akad. Wet. 51, 793 (1948).

[2] V. M. Mostepanenko and N. N. Trunov, The Casimir Effect and Its Applications (Oxford Science Publications, New York, 1997).

[3] J. Schwinger, Proc. Natl. Acad. Sci. U.S.A. 89, 4091 (1992); 89, 11118 (1992); 90, 958 (1993); 90, 2105 (1993); 90, 4505 (1993); 90, 7285 (1993); 91, 6473 (1994).

[4] R. Löstedt, B. P. Barber, R. A. Hiller, and S. J. Putterman, Phys. Rep. 281, 65 (1997).

[5] K. A. Milton and Y. J. Ng, hep-th/9607186.

[6] K. A. Milton and Y. J. Ng, Phys. Rev. E 57, 5504 (1998).

[7] I. Brevik, V. N. Marachevsky, and K. A. Milton, Phys. Rev. Lett. 82, 3948 (1999).

[8] J. S. Hoye and I. Brevik, quant-ph/0008088.

[9] M. Bordag, K. Kirsten, and D. Vassilevich, Phys. Rev. D 59, 085011 (1999).

[10] G. Lambiase, G. Scarpetta, and V. V. Nesterenko, hep-th/9912176.

[11] C. Molina-Paris and Matt Visser, Phys. Rev. D 56, 6629 (1997).

[12] C. E. Carlson, C. Molina-Paris, J. Perez-Mercader, and M. Visser, Phys. Rev. D 56, 1262 (1997).

[13] S. Liberati, M. Visser, F. Belgiorno, and D. W. Sciama, Phys. Rev. D 61, 085024 (2000).
[14] S. Liberati, M. Visser, F. Belgiorno, and D. W. Sciama, Phys. Rev. D 61, 085023 (2000).

[15] V. V. Nesterenko and I. G. Pirozhenko, Pis'ma Zh. Éksp. Teor. Fiz. 67, 420 (1998) [JETP Lett. 67, 445 (1998)].

[16] P. Candelas, Ann. Phys. (N.Y.) 143, 241 (1982).

[17] I. Brevik and G. Einevoll, Phys. Rev. D 37, 2977 (1988).

[18] I. Brevik and R. Sollie, J. Math. Phys. 31, 1445 (1990).

[19] I. Brevik, H. Skurdal, and R. Sollie, J. Phys. A 27, 6853 (1994).

[20] I. Bervik and G. H. Nyland, Ann. Phys. (N.Y.) 230, 321 (1994).

[21] T. H. Boyer, Phys. Rev. 174, 1764 (1968).

[22] K. A. Milton, L. L. De Raad, and J. Schwinger, Ann. Phys. (N.Y.) 115, 388 (1978).

[23] H. Falomir and K. Rébora (unpublished).

[24] I. S. Gradshteyn and I. M. Ryzhik, Table of Integrals, Series and Products (Academic Press, New York, 1980).

[25] M. Abramowitz and I. A. Stegun, Handbook of Mathematical Functions (Dover Publications, New York, 1972).

[26] A. Romeo, Phys. Rev. D 52, 7308 (1995).

[27] R. Balian and C. Bloch, Ann. Phys. (N.Y.) 60, 401 (1970).

[28] J. D. Jackson, Classical Electrodiynamics, 2nd ed. (Wiley, New York, 1975). 